\title{
AN EXTENSION OF THE NEMHAUSER-TROTTER THEOREM TO GENERALIZED VERTEX COVER WITH APPLICATIONS*
}

\author{
REUVEN BAR-YEHUDA ${ }^{\dagger}$, DANNY HERMELIN ${ }^{\ddagger}$, AND DROR RAWITZ ${ }^{\S}$
}

\begin{abstract}
The Nemhauser-Trotter theorem provides an algorithm which is frequently used as a subroutine in approximation algorithms for the classical VERTEX COVER problem. In this paper we present an extension of this theorem so it fits a more general variant of VERTEx Cover, namely, the Generalized Vertex Cover problem, where edges are allowed not to be covered at a certain predetermined penalty. We show that many applications of the original Nemhauser-Trotter theorem can be applied using our extension to Generalized Vertex Cover. These applications include a $(2-2 / d)$-approximation algorithm for graphs of bounded degree $d$, a polynomial-time approximation scheme (PTAS) for planar graphs, a $(2-\lg \lg n / 2 \lg n)$-approximation algorithm for general graphs, and a $2 k$ kernel for the parameterized Generalized Vertex Cover problem.
\end{abstract}

Key words. approximation algorithms, generalized vertex cover, local ratio technique, Nemhauser-Trotter theorem

AMS subject classifications. 68W25, 05C85, 68W40, 90C27

DOI. $10.1137 / 090773313$

1. Introduction. Given a graph $G=(V, E)$ with vertex weights, the classical VerTex COVER problem asks to find a minimum weight subset of vertices $S \subseteq V$ that covers all edges in $G$, i.e., a subset $S$ with $S \cap e \neq \emptyset$ for all $e \in E$. The VERTEX Cover problem is one of the most well studied problems in theoretical computer science and discrete mathematics in general, dating back to König's classical early 1930s result [20] and probably even prior to that. In 1972, Karp listed the decision version of VERTEX COVER in his famous list of initial 21 NP-complete problems [18].

One of the most well known results about VERTEX COVER is the half-integrality of the LP-relaxation of the standard integer programming formulation of VERTEX Cover (see, e.g., [22]). This result directly implies a 2-approximation algorithm for vertex cover (as observed by Hochbaum [14]). In 1975, only three years after the publication of Karp's famous NP-complete list, Nemhauser and Trotter published their seminal paper [23] in which they present a reduction that reduces the problem of finding a vertex cover in an arbitrary graph $G$ to that of finding a vertex cover in a subgraph of $G$ whose total weight is not much more than the weight of any of its vertex covers. This reduction is based on the half-integrality of VERTEX COVER, and it adds additional structure to the VERTEX COVER problem in general. Indeed, after applying the Nemhauser-Trotter reduction, one can use the total weight of the graph as a yardstick for analyzing approximate solutions rather than use the weight of the optimal solution of which there is rarely any knowledge. Below is a precise statement of the Nemhauser-Trotter theorem:

\footnotetext{
* Received by the editors October 9, 2009; accepted for publication January 12, 2010; published electronically March 17, 2010. An extended abstract was presented at the 7th Workshop on Approximation and Online Algorithms (WAOA), 2009.

http://www.siam.org/journals/sidma/24-1/77331.html

$\dagger$ Department of Computer Science, Technion IIT, Haifa 32000, Israel (reuven@cs.technion.ac.il).

${ }^{\ddagger}$ Department of Computer Science, University of Haifa, Haifa 31905, Israel (danny@cri.haifa.ac.il). Supported by the Adams Fellowship of the Israel Academy of Sciences and Humanities.

§School of Electrical Engineering, Tel-Aviv University, Tel-Aviv 69978, Israel (rawitz@eng.tau.ac. il).
} 
TheOREm 1 (Nemhauser and Trotter [23]). Let $(G, w)$ be an instance of VeRTEX Cover, with $G=(V, E)$ and $w: V \rightarrow \mathbb{Q}^{\geq 0}$. Then there is a polynomial-time algorithm that partitions the vertices of $G$ into three subsets, $V_{1}, V_{0}$, and $V_{1 / 2}$, such that

(i) if $S_{1 / 2}$ is an $\alpha$-approximate solution for $\left(G\left[V_{1 / 2}\right], w\right)$, then $V_{1} \cup S_{1 / 2}$ is an $\alpha$ approximate solution for $(G, w)$ for all $\alpha \geq 1$, and

(ii) the $w$-weight of any vertex cover in $G\left[V_{1 / 2}\right]$ is at least $\frac{1}{2} \sum_{v \in V_{1 / 2}} w(v)$.

The first condition of the theorem implies that we can restrict our attention to $G\left[V_{1 / 2}\right]$, ignoring vertices of $V_{1}$ and $V_{0}$ in $G$. The second condition of the theorem implies that finding a vertex cover of $G\left[V_{1 / 2}\right]$ that is properly contained in $V_{1 / 2}$ is guaranteed to give a vertex cover of $G$ whose weight is strictly less than twice the optimum.

It is important to note that the vertex sets $V_{0}, V_{1 / 2}$, and $V_{1}$ correspond to the values given to the variables by some half-integral optimal solution $x^{*}$ to the LPrelaxation of vertex cover. Namely, $V_{i}=\left\{u: x_{u}^{*}=i\right\}$ for every $i \in\{0,1 / 2,1\}$. It is not hard to verify that property (ii) of Theorem 1 follows directly from the half-integrality of the optimal solution $x^{*}$. Moreover, property (i) holds for every LP-based approximation algorithm since the optimal fraction solution of $G\left[V_{1 / 2}\right]$ is $(1 / 2, \ldots, 1 / 2)$. Nemhauser and Trotter [23] proved that taking $V_{1}$ into the solution is a local optimization step, namely, that even a non-LP-based approximation algorithm may be used to augment $V_{1}$.

The Nemhauser-Trotter theorem is an essential part of Hochbaum's $\left(2-\frac{2}{d}\right)$ approximation algorithm for graphs of bounded degree $d[15]$ and of the (2$\left.\frac{\lg \lg n}{2 \lg n}\right)$-approximation algorithm for general graphs given in [3]. In fact, many known approximation algorithms for VERTEX COVER and its special cases use the Nemhauser-Trotter theorem as a subroutine. Two other good examples are the polynomial-time approximation schemes (PTASs) of Lipton and Tarjan [21] and Baker [1] for VerTex Cover in planar graphs, ${ }^{1}$ where one finds an optimal solution in a large fraction of the graph and adds all remaining vertices to get a solution for the entire graph. We mention also Chen, Kanj, and Jia [8] who observed that the Nemhauser-Trotter theorem gives a $2 k$ kernel for the parameterized variant of Vertex Cover in the parameterized complexity setting when the parameter taken is the total weight of the required vertex cover (see also [9]).

In this paper we focus on a natural generalization of VERTEX COVER, which can be thought of as the prize-collecting version of the problem. In this variant, each edge in the given graph is allowed to be left uncovered at a certain predetermined penalty. Thus, the input now consists of a graph with vertex and edge weights, and the goal is to minimize the total weight of vertices selected to a solution plus the total weight of edges not covered by the solution. Observe that this is in fact a generalization of VERTEX COVER, since we return to the original problem by setting all edge weights to $\infty$. We call this generalization of Vertex Cover the Generalized Vertex Cover problem.

Generalized Vertex Cover:

Instance: A graph $G=(V, E)$ and a weight function $w: V \cup E \rightarrow$ $\mathbb{Q}^{\geq 0}$.

Solution: A subset $S$ of $V$.

Measure: $\operatorname{cost}(S)=\sum_{v \in S} w(v)+\sum_{e \in E, e \cap S=\emptyset} w(e)$.

\footnotetext{
${ }^{1}$ Baker's algorithm [1] originally did not use the Nemhauser-Trotter theorem, but adding it as a preprocessing step makes the analysis somewhat simpler.
} 
The first to consider Generalized Vertex Cover was Hochbaum [16], who provided a 2-approximation algorithm and also pointed out that GENERALIZED VERTEX COVER is polynomial-time solvable in bipartite graphs. The time complexity of this algorithm was later improved in [4], where a $d$-approximation algorithm for GENERALIzed VerTex Cover in $d$-hypergraphs was given as well. Hassin and Levin [12] studied a problem that extends Generalized Vertex Cover to one in which one pays a penalty for not covering an edge and a smaller penalty for covering an edge only by one of its endpoints. They presented a 2 -approximation algorithm for this problem. We further note that other prize-collecting covering problems were also studied extensively in the literature. This includes the paper by Hassin and Tamir [13] who considered the prize-collecting variant of the FACILITy LOCATION ON THE REAL Line problem, and the work of Goemans and Williamson [11] who presented approximation algorithms for the prize-collecting versions for Triangle InEQuality Traveling Salesman and Steiner Tree. See also [7, 17] for other prize-collecting facility location problems.

Due to the importance that the Nemhauser-Trotter theorem plays in designing approximation algorithms for VERTEX COVER and its special cases, a natural question to ask is whether a similar theorem can be found for Generalized Vertex Cover. Observe that the theorem does not carry on immediately to the more general case due to the different way that the edges now come into play; in fact, this poses a difficulty even in stating the theorem for the more general case. The main result of this paper overcomes these difficulties and gives an affirmative answer to the question above by proving a slightly different variant of the Nemhauser-Trotter theorem, which is essentially the same for most algorithmic applications. The following is a precise statement of our result.

Theorem 2. Let $(G, w)$ be an instance of Generalized Vertex Cover, with $G=(V, E)$ and $w: V \cup E \rightarrow \mathbb{Q}^{\geq 0}$. Then there is a polynomial-time algorithm that partitions the vertices of $G$ into three subsets, $V_{1}, V_{0}$, and $V_{1 / 2}$, and constructs another weight function $\widetilde{w}: V \cup E \rightarrow \mathbb{Q}^{\geq 0}$ such that

(i) if $S_{1 / 2}$ is an $\alpha$-approximate solution for $\left(G\left[V_{1 / 2}\right], \widetilde{w}\right)$, then $V_{1} \cup S_{1 / 2}$ is an $\alpha$ approximate solution for $(G, w)$ for all $\alpha \geq 1$, and

(ii) the $\widetilde{w}$-cost of any subset $S \subseteq V_{1 / 2}$ is at least $\frac{1}{2} \sum_{v \in V_{1 / 2}} \widetilde{w}(v)$.

Observe the difference in the second condition of the theorem which is necessary since any subset of vertices is a potential solution in Generalized VerTex Cover. This is what makes the proof of the theorem in the generalized case more challenging. Another challenge is that as the edges in Generalized Vertex Cover play a different role, we are not guaranteed the combinatorial structure provided by the original theorem. For instance, in the original theorem the subset $V_{0}$ in the partition had to be an independent set, as otherwise any vertex cover had to include at least one vertex from $V_{0}$. In our case we can never require such a condition; we must assume that there can be an edge between any pair of vertices in $V_{0}$. Furthermore, in the original theorem, it is clear that $V_{1}$ must separate $V_{0}$ from $V_{1 / 2}$ in $G$. Again, in our case this is not necessarily so, which makes the reduction more difficult since we insist that the resulting subgraph be induced, i.e., obtained only by deleting vertices, a fact that allows carrying on hereditary properties of $G$ through the reduction.

With the help of Theorem 2, we can show that many algorithms for VERTEX Cover which use the Nemhauser-Trotter theorem as a subroutine can be modified so that they apply also for the more general case. In particular, we obtain the following 
new results for Generalized Vertex Cover as almost immediate corollaries of Theorem 2:

1. a $(2-2 / d)$-approximation algorithm for graphs of bounded degree $d$,

2. a PTAS for planar graphs,

3. a $(2-\lg \lg n / 2 \lg n)$-approximation algorithm for general graphs,

4. a $2 k$ kernel for the parameterized version of Generalized VerTex Cover.

The reader should not be misled into thinking our results imply that VERTEX Cover and Generalized Vertex Cover are in fact the same in any graph class. To see that this is not so, note that while VERTEx Cover is polynomial-time solvable in complete graphs, Generalized VerTex Cover in complete graphs is essentially as hard to approximate as VERTEX COVER in any general graph (and thus it cannot be approximated within $10 \sqrt{5}-21 \approx 1.36$, unless $\mathrm{P}=\mathrm{NP}[10]$ ). This can be seen by the following reduction from VerTex Cover in general graphs to Generalized Vertex Cover in complete graphs: Given a graph $G$, transform $G$ into a complete graph $G^{\prime}$ by adding all necessary edges, and assign a weight to these edges such that their total weight is substantially smaller than the weight of any vertex in the graph. All original edges are assigned a weight of $\infty$, and the vertex weights remain the same. It is not difficult to see that any $\alpha$-approximate vertex cover for $G$ is also an $(\alpha+\epsilon)$-approximate generalized vertex cover of $G^{\prime}$ for any $\epsilon>0$ as small as we want and vice versa.

Our work is also related to the recent work of Könemann, Parekh, and Segev [19], who presented a reduction from partial covering to prize-collecting covering, or in our context, from Partial Vertex Cover to Generalized Vertex Cover. The Partial Vertex Cover problem is another natural generalization of VerTEX COVER, where now the goal is to find a minimum weight subset of vertices that covers a prespecified number of edges in the graph. Könemann, Parekh, and Segev [19] showed how to transform a specific class of $\alpha$-approximation algorithms for Generalized Vertex Cover into $\left(\frac{4}{3} \alpha+\epsilon\right)$-approximation algorithms for Partial Vertex Cover. The algorithm in our Theorem 2 can be used in conjunction with an algorithm from this specific class that works under the assumption that the cost of any generalized vertex cover is at least half of the total weight of the vertices, and so Theorem 2 combined with the work of Könemann, Parekh, and Segev gives a more refined reduction from Partial Vertex Cover to Generalized Vertex Cover.

The rest of the paper is devoted to proving Theorem 2 along with all of its applications mentioned above. In the next section, we discuss some preliminaries necessary for our proof, and in particular, we review the local-ratio method which plays an important part in many of our results. In section 3 we provide all details of the proof of Theorem 2, and in section 4 we discuss all applications mentioned above.

2. Preliminaries. In this section we discuss notation and previous work that is necessary for presenting our results. In particular, we introduce terminology that is used for proving Theorem 2 and briefly review the local-ratio technique which we use throughout the paper.

We consider throughout the paper graphs that have weights assigned to their vertices and edges. Let $G=(V, E)$ be a graph given along with a weight function $w: V \cup E \rightarrow \mathbb{Q}^{\geq 0}$. For any edge $\{u, v\} \in E$, we write $w(u, v)$ as a shorthand for $w(\{u, v\})$. For a subset of vertices $S \subseteq V$, we let $w(S)=\sum_{v \in S} w(v)$, and for a pair of subsets $S_{1}, S_{2} \subseteq V$, we use $w\left(S_{1}, S_{2}\right)$ for the weight of edges with one endpoint in 
$S_{1}$ and one endpoint in $S_{2}$ (edges whose endpoints are in $S_{1} \cap S_{2}$ are counted only once); namely,

$$
w\left(S_{1}, S_{2}\right)=\sum_{u \in S_{1}} \sum_{v \in S_{2}} w(u, v)-\sum_{\{u, v\} \subseteq S_{1} \cap S_{2}} w(u, v) .
$$

Note that $w(S, S)$ stands for the total weight of edges in $G[S]$. Observe that the cost of a subset $S \subseteq V$ in $G$ is

$$
\operatorname{cost}(S)=w(S)+w(V \backslash S, V \backslash S)
$$

Let $\operatorname{opt}(G, w)$ denote the cost of the optimal generalized vertex cover in $(G, w)$. For an $\alpha>0$, we say that $S$ is $\alpha$-approximate if $\operatorname{cost}(S) \leq \alpha \cdot \operatorname{opt}(G, w)$. Also, we call any subset $S \subseteq V$ feasible if it has cost less than $\infty$.

The local-ratio technique [3] is central in our proof of Theorem 2 and is also used in its applications. The technique in most part is based on the local-ratio lemma, which in our terms can be stated as follows.

Lemma 1 (local-ratio [3]). Let $\left(G, w_{1}\right)$ and $\left(G, w_{2}\right)$ be two instances of GenERALIzed Vertex Cover, with $G=(V, E)$ a graph and $w_{1}, w_{2}: V \cup E \rightarrow \mathbb{Q}^{\geq 0}$ two weight functions. If $S \subseteq V$ is an $\alpha$-approximate solution both in $\left(G, w_{1}\right)$ and in $\left(G, w_{2}\right)$, then $S$ is also an $\alpha$-approximate solution in $\left(G, w_{1}+w_{2}\right)$.

A typical local-ratio algorithm is recursive. In each recursive step, it defines a weight function $w_{1}$ in such a way that $w_{2}=w-w_{1}$ still assigns nonnegative weights to all elements, and at least one element with nonzero weight with respect to $w$ gets zero weight with respect to $w_{2}$. The algorithm then recursively solves the instance of the problem with $w_{2}$ as the given weight function and fixes the returned solution, so it is a good approximate solution with respect to both $w_{1}$ and $w_{2}$. By the local-ratio lemma, this solution is guaranteed to be a good approximation with respect to $w$ as well. The following definition hints on how to select a good weight function.

Definition 1 ( $\alpha$-effectiveness [2]). A weight function $w_{1}$ is said to be $\alpha$-effective in $G$ if the following holds: If a subset of vertices is feasible, then it is also $\alpha$ approximate with respect to $w_{1}$.

Below we give a variation of the local-ratio lemma which uses the notion of $\alpha$ effectiveness and is the variation that will actually be used in the paper. Its proof is immediate from the local-ratio lemma and the definition of $\alpha$-effectiveness and is left to the reader.

Lemma 2. Let $(G, w)$ be an instance of Generalized Vertex Cover, and let $w_{\varepsilon}$ be a weight function which is $\alpha$-effective in $G$. If $S$ is a $\beta$-approximate solution for $\left(G, w-w_{\varepsilon}\right)$, then $S$ is a $\max \{\alpha, \beta\}$-approximate solution for $(G, w)$.

3. The main proof. In this section we present the central result of this paper, namely, the proof of Theorem 2. Our proof consists of two main steps: In the first, similar to the proof of the original Nemhauser-Trotter theorem, we obtain an initial partition of the vertices of our graph $G$ into three classes according to an optimal solution for an appropriate bipartite graph constructed from $G$. However, unlike the original proof, in our case we can have edges between all classes and inside each class. We show that the only really problematic edges are those that are between two particular classes. These edges are taken care of in the second step by several applications of the local-ratio lemma, at the end of which we obtain our desired partition of the vertices of $G$ and the desired weight function $\widetilde{w}$. Before describing both steps in actual detail, we start with the following lemma which will later be used in our proof but is also of independent interest. 
Lemma 3. Generalized Vertex Cover is polynomial-time solvable in bipartite graphs.

Proof. Let $B=\left(V, V^{\prime}, F\right)$ be a bipartite graph, and let $w: V \cup V^{\prime} \cup F \rightarrow \mathbb{Q}^{\geq 0}$ be a weight function. Construct a flow-network $N$ from $B$ by adding to $B$ a source $s$ and a destination $t$, with $s$ connected to all vertices in $V$ and vertices in $V^{\prime}$ connected to $t$. Define the capacities of the edges in $N$ by the following:

- $c(s, v)=w(v)$ for all $v \in V$,

- $c(u, v)=w(u, v)$ for all $\{u, v\} \in F$, and

- $c(v, t)=w(v)$ for all $v \in V^{\prime}$.

Observe that there is a one-to-one correspondence between edges in $B$ and $s, t$ paths in $N$ and that the edges on an $s, t$-path in $N$ correspond to the three ways of covering the corresponding edge in $B$ : either adding one of its endpoints to the generalized vertex cover or not covering this edge at all. Specifically, given a subset $U \subseteq V \cup V^{\prime}$, the corresponding $s, t$-cut is $(S, T)$, where $S=\{s\} \cup(V \backslash U) \cup\left(V^{\prime} \cap U\right)$ and $T=\{t\} \cup\left(V \cup V^{\prime}\right) \backslash S$, and conversely, given an $s, t$-cut $(S, T)$, the corresponding cover is $U=\left(S \cap V^{\prime}\right) \cup(T \cap V)$ (see Figure 1). Hence, there is a one-to-one correspondence between generalized vertex covers in $B$ and $s, t$-cuts in $N$. Moreover, by our selection of capacities, each generalized vertex cover corresponds to an $s, t$-cut whose capacity is equal to the cost of the cover. Since one can compute minimum $s, t$-cuts by standard flow techniques, the lemma is proven.
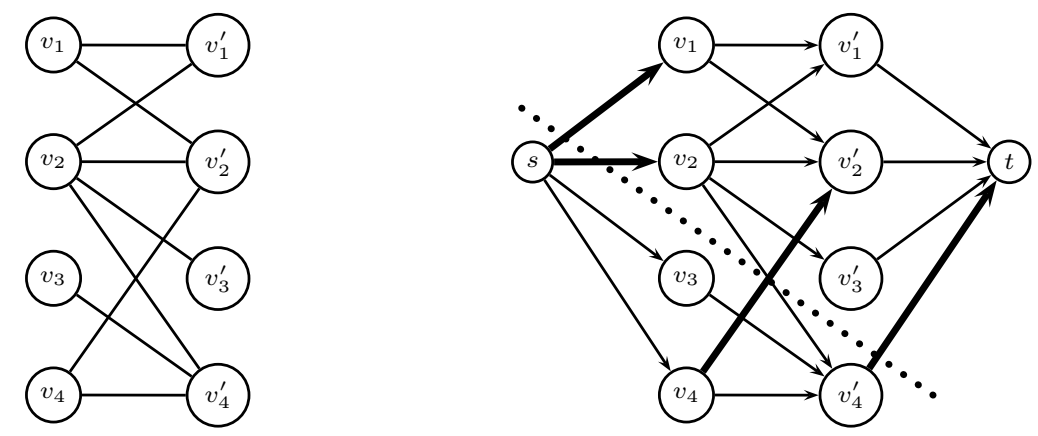

FIG. 1. A bipartite graph and the corresponding network: The dotted line represents an $s, t$-cut; the thick edges that cross the $s, t$-cut correspond to the cover $\left\{v_{1}, v_{2}, v_{4}^{\prime}\right\}$.

3.1. Step I. Given an instance $(G, w)$ for Generalized Vertex Cover, with $G=(V, E)$ and $w: V \cup E \rightarrow \mathbb{Q}^{\geq 0}$, we construct a bipartite graph $B=\left(V, V^{\prime}, F\right)$ along with a weight function $w_{B}: V \cup V^{\prime} \cup F \rightarrow \mathbb{Q}^{\geq 0}$ for $B$, as follows: The set $V^{\prime}$ contains a duplicate vertex for each vertex in $V$ and is defined by $V^{\prime}=\left\{v^{\prime} \mid v \in V\right\}$. The set $F$ of edges in $B$ includes the pair of edges $\left\{u, v^{\prime}\right\}$ and $\left\{u^{\prime}, v\right\}$ for each edge $\{u, v\} \in E$. We define $w_{B}$ by $w_{B}(v), w_{B}\left(v^{\prime}\right)=w(v)$ for all $v, v^{\prime} \in V \cup V^{\prime}$ and by $w_{B}\left(u^{\prime}, v\right), w_{B}\left(u, v^{\prime}\right)=w(u, v)$ for all $\left\{u, v^{\prime}\right\},\left\{u^{\prime}, v\right\} \in F$. Here, and throughout the remainder of this section, we denote by $S^{\prime}$ the set of duplicates of some subset $S \subseteq V$. That is, $S^{\prime}=\left\{v^{\prime} \mid v \in S\right\}$.

We next compute an optimal solution $S_{B}^{*}$ in $B$ using the algorithm implied by Lemma 3. According to the computed solution $S_{B}^{*}$, we partition $V$ into the following three subsets:

$$
U_{1}=\left\{v \mid v, v^{\prime} \in S_{B}^{*}\right\}, \quad U_{0}=\left\{v \mid v, v^{\prime} \notin S_{B}^{*}\right\}, \quad \text { and } \quad U_{1 / 2}=V \backslash\left(U_{1} \cup U_{0}\right) .
$$


We first show that we may assume that $S_{B}^{*}$ does not include any vertices of $U_{1 / 2}^{\prime}$, namely, that $S_{B}^{*} \cap U_{1 / 2}^{\prime}=\emptyset$.

Claim 1. $\operatorname{cost}_{B}\left(U_{1} \cup U_{1}^{\prime} \cup U_{1 / 2}\right) \leq \operatorname{cost}_{B}\left(S_{B}^{*}\right)$.

Proof. By a simple manipulation of the cost of $S_{B}^{*}$ in $\left(B, w_{B}\right)$, we get

$$
\begin{aligned}
\operatorname{cost}_{B}\left(S_{B}^{*}\right) & =w_{B}\left(S_{B}^{*}\right)+w_{B}\left(U_{0} \cup U_{1 / 2} \backslash S_{B}^{*}, U_{0}^{\prime} \cup U_{1 / 2}^{\prime} \backslash S_{B}^{*}\right) \\
& \geq w_{B}\left(S_{B}^{*}\right)+w_{B}\left(U_{0}, U_{0}^{\prime}\right)+w_{B}\left(U_{0}, U_{1 / 2}^{\prime} \backslash S_{B}^{*}\right)+w_{B}\left(U_{0}^{\prime}, U_{1 / 2} \backslash S_{B}^{*}\right) .
\end{aligned}
$$

Thus, since $w_{B}\left(U_{0}, U_{1 / 2}^{\prime} \backslash S_{B}^{*}\right)+w_{B}\left(U_{0}^{\prime}, U_{1 / 2} \backslash S_{B}^{*}\right)=w_{B}\left(U_{0}, U_{1 / 2}^{\prime}\right)$, we have

$$
\operatorname{cost}_{B}\left(S_{B}^{*}\right) \geq w_{B}\left(S_{B}^{*}\right)+w_{B}\left(U_{0}, U_{0}^{\prime}\right)+w_{B}\left(U_{0}, U_{1 / 2}^{\prime}\right)=\operatorname{cost}_{B}\left(U_{1} \cup U_{1}^{\prime} \cup U_{1 / 2}\right),
$$

and the claim is proven.

Next, using the simple observation given by Claim 1, we show that we are on the right direction with our initial partition of the vertices of $G$, since there is an optimal solution which includes all vertices of $U_{1}$ and no vertex of $U_{0}$.

Claim 2. There is an optimal solution $S$ for $(G, w)$, with $U_{1} \subseteq S$ and $U_{0} \cap S=\emptyset$.

Proof. Let $S$ be any subset of vertices in $G$, and let $S_{z}=U_{z} \cap S$ for $z \in\{1,1 / 2,0\}$. Also, let $T=V \backslash S$ and $T_{z}=U_{z} \backslash S_{z}$ for $z \in\{1,1 / 2,0\}$. To prove the claim, we argue that the solution $U_{1} \cup S_{1 / 2}$ does not have greater cost than $S$. The claim then immediately follows by taking $S$ to be optimal.

For this, consider the difference between the cost of $S$ and the cost of $U_{1} \cup S_{1 / 2}$. The only advantage the former has over the latter is that it does not pay for any vertex in $T_{1}$ nor for any edge between $S_{0}$ and $U_{0} \cup T_{1 / 2}$-all elements which are paid for by $U_{1} \cup S_{1 / 2}$. However, $S$ has to pay for all vertices in $S_{0}$ and all edges between $T_{1}$ and $T$, while $U_{1} \cup S_{1 / 2}$ does not. (See depiction in Figure 2a.) Since this is the only difference between the two solutions, we have

$$
\operatorname{cost}_{G}(S)-\operatorname{cost}_{G}\left(U_{1} \cup S_{1 / 2}\right)=w\left(S_{0}\right)+w\left(T_{1}, T\right)-w\left(T_{1}\right)-w\left(S_{0}, U_{0} \cup T_{1 / 2}\right) .
$$

Now, let us construct a solution $S_{B}$ for the bipartite graph $B=B(G)$ described above, defined by $S_{B}=\left(V \backslash T_{0}\right) \cup S_{1}^{\prime}$, and let us compare this solution to $S_{B}^{*}$. Claim 2 implies that there is no loss of generality in assuming that $S_{B}^{*}=U_{1} \cup U_{1 / 2} \cup U_{1}^{\prime}$, i.e., that $S_{B}^{*}$ does not include any vertices of $U_{1 / 2}^{\prime}$. Now $S_{B}$ does not pay for any vertex in $T_{1}^{\prime}$, while $S_{B}^{*}$ does, nor does it pay for any edges between $S_{0}$ and $U_{0}^{\prime} \cup U_{1 / 2}^{\prime}$, all of which are paid for by $S_{B}^{*}$. On the other hand, $S_{B}^{*}$ does not pay for any vertex

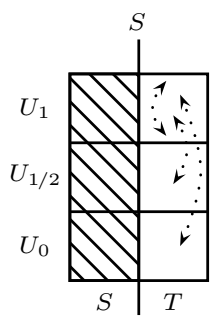

(a)

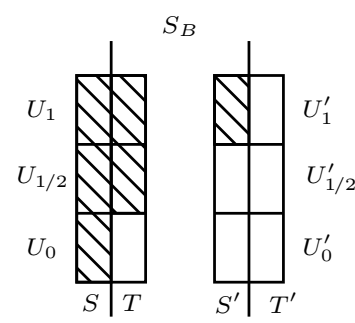

(b)

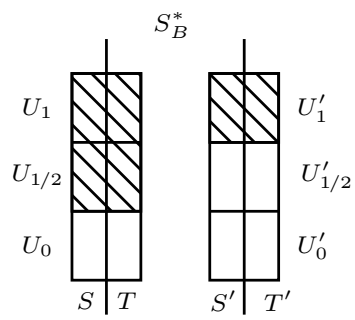

FIG. 2. Depiction of differences between (a) $S$ and $U_{1} \cup S_{1 / 2}$ and (b) between $S_{B}$ and $S_{B}^{*}$; the dotted lines correspond to edges that are not covered by one cover but are covered by the other. 
in $S_{0}$ nor for any edge between $T_{1}^{\prime}$ and $T_{0}$. (See depiction in Figure 2b.) Noting that this is the exact difference between their costs and that all weights are positive, we get

$$
\begin{aligned}
\operatorname{cost}_{B}\left(S_{B}\right)-\operatorname{cost}_{B}\left(S_{B}^{*}\right)= & w_{B}\left(S_{0}\right)+w_{B}\left(T_{1}^{\prime}, T_{0}\right)-w_{B}\left(T_{1}^{\prime}\right)-w_{B}\left(S_{0}, U_{0}^{\prime} \cup U_{1 / 2}^{\prime}\right) \\
= & w\left(S_{0}\right)+w\left(T_{1}, T_{0}\right)-w\left(T_{1}\right)-w\left(S_{0}, U_{0} \cup U_{1 / 2}\right) \\
= & w\left(S_{0}\right)+w\left(T_{1}, T\right)-w\left(T_{1}, T_{1} \cup T_{1 / 2}\right) \\
& -w\left(T_{1}\right)-w\left(S_{0}, U_{0} \cup T_{1 / 2}\right)-w\left(S_{0}, S_{1 / 2}\right) \\
= & \operatorname{cost}_{G}(S)-\operatorname{cost}_{G}\left(U_{1} \cup S_{1 / 2}\right) \\
& -w\left(T_{1}, T_{1} \cup T_{1 / 2}\right)-w\left(S_{0}, S_{1 / 2}\right) \\
\leq & \operatorname{cost}_{G}(S)-\operatorname{cost}_{G}\left(U_{1} \cup S_{1 / 2}\right)
\end{aligned}
$$

As $S_{B}^{*}$ is optimal in $B$, we know that $\operatorname{cost}_{B}\left(S_{B}\right)-\operatorname{cost}_{B}\left(S_{B}^{*}\right) \geq 0$. Hence, $\operatorname{cost}(S)-$ $\operatorname{cost}\left(U_{1} \cup S_{1 / 2}\right) \geq 0$, and so the claim is proven.

Claim 2 implies that we can safely restrict ourselves to solutions for $(G, w)$ which include all vertices of $U_{1}$ and no vertex of $U_{0}$. Therefore, all edges which have at least one endpoint in $U_{1}$ are redundant to us in this sense. Also, edges with both endpoints in $U_{0}$ are redundant, since we can safely leave these uncovered. The same is not true for edges between $U_{0}$ and $U_{1 / 2}$, as these still might need to be covered. We take care of these edges in the second step of our algorithm, but for now consider the graph $H$ obtained by deleting all edges between vertices of $U_{0}$ in the induced subgraph $G\left[U_{0} \cup U_{1 / 2}\right]$ of $G$. Define a weight function $w_{H}$ for $H$ which equals $w$ on all edges of $H$ and all vertices of $U_{1 / 2}$ and assigns $\infty$ to all vertices in $U_{0}$. In the following we argue that a good approximation for $\left(H, w_{H}\right)$ gives a good approximation for $(G, w)$.

Claim 3. If $S$ is $\alpha$-approximate for $\left(H, w_{H}\right)$, then $U_{1} \cup S$ is $\alpha$-approximate for $(G, w)$.

Proof. Let $S_{H}^{*}$ denote an optimal solution in $\left(H, w_{H}\right)$, and assume without loss of generality that $\operatorname{cost}_{H}\left(S_{H}^{*}\right)<\infty$. Then $\operatorname{cost}_{H}(S) \leq \alpha \cdot \operatorname{cost}_{H}\left(S_{H}^{*}\right) \leq \infty$. Now, according to Claim 2, there is an optimal solution for $(G, w)$ which includes all vertices of $U_{1}$ and no vertex of $U_{0}$, so let $S^{*}$ be such a solution, with $S_{1 / 2}^{*}=S^{*} \cap U_{1 / 2}$ and $T_{1 / 2}^{*}=U_{1 / 2} \backslash S_{1 / 2}^{*}$. Hence,

$$
\begin{aligned}
\operatorname{cost}_{G}\left(U_{1} \cup S\right) & =w\left(U_{1}\right)+w\left(U_{0}, U_{0}\right)+\operatorname{cost}_{H}(S) \\
& \leq w\left(U_{1}\right)+w\left(U_{0}, U_{0}\right)+\alpha \cdot \operatorname{cost}_{H}\left(S_{H}^{*}\right) \\
& \leq \alpha \cdot\left(w\left(U_{1}\right)+w\left(U_{0}, U_{0}\right)+\operatorname{cost}_{H}\left(S_{1 / 2}^{*}\right)\right) \\
& =\alpha \cdot\left(w\left(U_{1}\right)+w\left(U_{0}, U_{0}\right)+w_{H}\left(S_{1 / 2}^{*}\right)+w_{H}\left(U_{0} \cup T_{1 / 2}^{*}, U_{0} \cup T_{1 / 2}^{*}\right)\right) \\
& =\alpha \cdot\left(w\left(U_{1}\right)+w\left(U_{0}, U_{0}\right)+w\left(S_{1 / 2}^{*}\right)+w\left(U_{0} \cup T_{1 / 2}^{*}, U_{0} \cup T_{1 / 2}^{*}\right)\right) \\
& =\alpha \cdot \operatorname{cost}_{G}\left(S^{*}\right)
\end{aligned}
$$

and the claim is proven.

Furthermore, we show that the total weight of elements in $H$ with finite weight is at most twice the cost of any solution of $\left(H, w_{H}\right)$.

Claim 4. $w_{H}\left(U_{1 / 2}\right)+w_{H}\left(U_{0}, U_{1 / 2}\right) \leq 2 \cdot \operatorname{cost}_{H}(S)$ for every $S \subseteq U_{0} \cup U_{1 / 2}$.

Proof. If $S \nsubseteq U_{1 / 2}$, then $\operatorname{cost}_{H}(S)=\infty$, and the claim is trivial. Assume, therefore, that $S \subseteq U_{1 / 2}$, and denote $T=U_{1 / 2} \backslash S$. Consider the solution $S_{B}=$ $U_{1} \cup U_{1}^{\prime} \cup S \cup S^{\prime}$ for the bipartite graph $B$ constructed above. The cost of this 
solution is

$$
\begin{aligned}
\operatorname{cost}_{B}\left(S_{B}\right) & =w_{B}\left(U_{1} \cup U_{1}^{\prime}\right)+w_{B}\left(S \cup S^{\prime}\right)+w_{B}\left(U_{0} \cup T, U_{0}^{\prime} \cup T^{\prime}\right) \\
& =2 \cdot w\left(U_{1}\right)+2 \cdot w(S)+w\left(U_{0}, U_{0}\right)+w(T, T)+2 \cdot w\left(U_{0}, T\right) \\
& =2 \cdot w\left(U_{1}\right)+2 \cdot \operatorname{cost}_{H}(S)+w\left(U_{0}, U_{0}\right)-w(T, T) .
\end{aligned}
$$

Now let us compare this solution to $S_{B}^{*}$, the optimal solution of $B$. Recall that we can assume that $U_{1 / 2} \subseteq S_{B}^{*}$ and $S_{B}^{*} \cap U_{1 / 2}^{\prime}=\emptyset$. The cost of $S_{B}^{*}$ equals the total weight of its vertices plus the total weight of all edges between $U_{0}$ and $U_{0}^{\prime} \cup U_{1 / 2}^{\prime}$. We have

$$
\operatorname{cost}_{B}\left(S_{B}^{*}\right)=2 \cdot w\left(U_{1}\right)+w\left(U_{1 / 2}\right)+w\left(U_{0}, U_{0}\right)+w\left(U_{0}, U_{1 / 2}\right) .
$$

The claim can now be easily proven by combining the two equalities above with the fact that $\operatorname{cost}_{B}\left(S_{B}^{*}\right) \leq \operatorname{cost}_{B}\left(S_{B}\right)$.

3.2. Step II. Note that while the instance $\left(H, w_{H}\right)$ is close to what we aimed to achieve, it is not quite there. One reason is that $H$ is not an induced subgraph of $G$; it contains vertices of $U_{0}$ without the edges between them. Another reason is that the $U_{0}$ vertices have $w_{H}$-weight equal to $\infty$, and therefore, any feasible solution for $\left(H, w_{H}\right)$ will not satisfy the second condition of Theorem 2. We cannot simply discard these $U_{0}$ vertices, since some of them might be connected to vertices in $U_{1 / 2}$. For this reason, we apply the local-ratio lemma to eliminate edges between $U_{0}$ and $U_{1 / 2}$. This is done by applying the following procedure that iteratively subtracts 1-effective weight functions from $w_{H}$, in order to obtain the weight function $\widetilde{w}$ promised by Theorem 2 .

While there is an edge $e_{0}=\{u, v\}$ in $H$ with $u \in U_{0}, v \in U_{1 / 2}$, and $w_{H}\left(e_{0}\right), w_{H}(v)>0$, do:

a. Let $\varepsilon=\min \left\{w_{H}\left(e_{0}\right), w_{H}(v)\right\}$.

b. Define the weight function $w_{\varepsilon}$ for $H$ by:

$-w_{\varepsilon}(v), w_{\varepsilon}\left(e_{0}\right)=\varepsilon$, and

- $w_{\varepsilon}(x), w_{\varepsilon}(e)=0$ for all $x \neq v$ and $e \neq e_{0}$.

c. $w_{H}=w_{H}-w_{\varepsilon}$.

The above procedure terminates in polynomial time, since at each iteration, either a vertex or an edge gets its $w_{H}$-weight reduced to zero. The weight function $\widetilde{w}$ is defined to be $w_{H}$ at the end of the procedure. We define the partition of the vertices in $G$ which is promised in Theorem 2 using $\widetilde{w}$ as follows:

$$
V_{1}=U_{1} \cup\left\{v \in U_{1 / 2} \mid \widetilde{w}(v)=0\right\}, \quad V_{0}=U_{0}, \quad \text { and } \quad V_{1 / 2}=V \backslash\left(V_{1} \cup V_{0}\right) .
$$

To complete the proof, we argue that a good approximation for $\left(G\left[V_{1 / 2}\right], \widetilde{w}\right)$ gives a good approximation for $\left(H, w_{H}\right)$ and that $w\left(V_{1 / 2}\right)$ is at most twice the cost of any solution for $\left(G\left[V_{1 / 2}\right], \widetilde{w}\right)$.

Claim 5. If $S$ is $\alpha$-approximate for $\left(G\left[V_{1 / 2}\right], \widetilde{w}\right)$, then $S \cup\{v \mid \widetilde{w}(v)=0\}$ is $\alpha$-approximate for $\left(H, w_{H}\right)$.

Proof. We prove the claim by using induction on the number of steps applied in this procedure. According to Lemma 2, it suffices to show that any weight function $w_{\varepsilon}$ subtracted in the procedure above is 1-effective. But this is immediate since any solution with cost less than $\infty$ in $\left(H, w_{\varepsilon}\right)$ has cost exactly $\varepsilon$ : It pays either for not covering the edge $e_{0}$ or for its endpoint in $U_{1 / 2}$, but never for both. Finally, since $G\left[V_{1 / 2}\right]$ is obtained by removing vertices from $H$ with either 0 or $\infty \widetilde{w}$-weights, an $\alpha$-approximation for $\left(G\left[V_{1 / 2}\right], \widetilde{w}\right)$ implies an $\alpha$-approximation for $(H, \widetilde{w})$, and the claim follows. 
Claim 6. $\widetilde{w}\left(V_{1 / 2}\right) \leq 2 \cdot \operatorname{cost}_{G\left[V_{1 / 2}\right]}(S)$ for every $S \subseteq V_{1 / 2}$.

Proof. By Claim 4, we have

$$
w_{H}\left(U_{1 / 2}\right)+w_{H}\left(U_{0}, U_{1 / 2}\right) \leq 2 \cdot \operatorname{cost}_{H}(S)
$$

for any $S \subseteq U_{0} \cup U_{1 / 2}$. Since at each iteration in the procedure above we subtract exactly $2 \varepsilon$ from each side of this inequality and at the end of which $G\left[V_{1 / 2}\right]$ includes all positive weighted vertices of $H$, we get that

$$
\widetilde{w}\left(V_{1 / 2}\right) \leq \widetilde{w}\left(U_{1 / 2}\right)+\widetilde{w}\left(U_{0}, U_{1 / 2}\right) \leq 2 \cdot \operatorname{cost}_{G\left[V_{1 / 2}\right]}(S),
$$

and the claim is proven.

The partition $V_{1}, V_{0}$, and $V_{1 / 2}$, along with the weight function $\widetilde{w}$, satisfies both conditions of Theorem 2. Combining Claim 5 with Claim 3 proves the first condition, while the second condition follows directly from Claim 6 .

4. Applications. As mentioned in section 1, the Nemhauser-Trotter theorem has several applications in designing approximation algorithms for VERTEx Cover. In the following we show that several of these extend to Generalized VERTEX Cover using Theorem 2. The section is divided into four parts, with each part giving a different corollary of Theorem 2 . We start with a $\left(2-\frac{2}{d}\right)$-approximation algorithm for graphs of bounded degree $d$ and then continue to show a PTAS for planar graphs and a $\left(2-\frac{\lg \lg n}{2 \lg n}\right)$-approximation algorithm for general graphs. Finally, we show that Theorem 2 gives a linear kernel for parameterized Generalized Vertex Cover.

4.1. Bounded degree graphs. Our first application for Theorem 2 is a $\left(2-\frac{2}{d}\right)$ approximation algorithm for Generalized VerTex COVER in graphs of bounded degree $d$. This is an analogous result to an algorithm of Hochbaum [15] that applies the original Nemhauser-Trotter theorem to obtain the same approximation ratio for VERTEX Cover in graphs of bounded degree $d$. In her algorithm, Hochbaum uses a classical graph-theoretic result by Brooks [6] which states that any graph of bounded degree $d$ which is not complete nor an odd cycle can be properly colored in $d$ colors. (That is, its vertex set can be partitioned into $d$ classes, with no edges between any pair of vertices in the same class.) Together with the Nemhauser-Trotter theorem, this is basically all that is necessary for Hochbaum's algorithm. Indeed, in our case it is also all that is necessary, due to the following lemma.

Lemma 4. Generalized Vertex Cover is polynomial-time solvable in cycles.

Proof. We first show how to solve the problem in paths using dynamic programming. Let $G$ be a path $v_{1}, \ldots, v_{n}$, and let $G_{i}$ denote the path $v_{1}, \ldots, v_{i}$ for all $i \in\{1, \ldots, n\}$. We compute two tables, $\Pi$ and $\Pi^{\prime}$, where $\Pi(i)$ is the cost of the optimal generalized vertex cover of $G_{i}$ that contains $v_{i}$, and $\Pi^{\prime}(i)$ is the cost of the optimal generalized vertex cover of $G_{i}$ that does not contain $v_{i}$. We have $\Pi(1)=w\left(v_{1}\right)$ and $\Pi^{\prime}(1)=0$, and we may compute $\Pi(i)$ for $i>1$ using the following recurrence:

$$
\begin{aligned}
\Pi(i) & =\min \left\{\Pi(i-1)+w\left(v_{i}\right), \Pi^{\prime}(i-1)+w\left(v_{i}\right)\right\} \\
\Pi^{\prime}(i) & =\min \left\{\Pi(i-1), \Pi^{\prime}(i-1)+w\left(v_{i-1}, v_{i}\right)\right\} .
\end{aligned}
$$

The cost of the optimal solution for the path $G$ is $\min \left\{\Pi(n), \Pi^{\prime}(n)\right\}$. Also, note that the computation of $\Pi(i)$ can be easily modified to output a corresponding optimal generalized vertex cover.

Next, we solve the problem in cycles using a reduction to the problem in paths. Given a weighted cycle $G$ on the vertices $v_{1}, \ldots, v_{n}$, we define $G_{1}$ to be the path 
$v_{0}, v_{1}, \ldots, v_{n}$, where $w\left(v_{0}\right)=\infty$ and $w\left(v_{0}, v_{1}\right)=w\left(v_{1}, v_{n}\right)$, and $G_{2}$ to be the path $v_{1}, \ldots, v_{n}, v_{n+1}$, where $w\left(v_{n+1}\right)=\infty$ and $w\left(v_{n}, v_{n+1}\right)=w\left(v_{1}, v_{n}\right)$. It is not hard to verify that $\operatorname{opt}(G)=\min \left\{\operatorname{opt}\left(G_{1}\right), \operatorname{opt}\left(G_{2}\right)\right\}$.

Corollary 3. $d$-Generalized Vertex Cover can be approximated within a factor of $2-\frac{2}{d}$ for any $d>1$.

Proof. Given an instance $(G, w)$ of Generalized Vertex Cover, with $G$ having no degree greater than $d$, apply Theorem 2 to obtain $V_{1}, V_{0}, V_{1 / 2}$, and $\widetilde{w}$. If $G\left[V_{1 / 2}\right]$ is a cycle, then an optimal generalized vertex cover in $\left(G\left[V_{1 / 2}\right], \widetilde{w}\right)$ can be found in polynomial time by Lemma 4 . If $G\left[V_{1 / 2}\right]$ is the complete graph, then $G\left[V_{1 / 2}\right]$ contains $O\left(d^{2}\right)=O(1)$ vertices, and we can trivially compute in polynomial time an optimal generalized vertex cover.

Assume, therefore, that $G\left[V_{1 / 2}\right]$ is neither complete nor a cycle, and let $S_{1 / 2}^{*}$ denote the optimal solution for $\left(G\left[V_{1 / 2}\right], \widetilde{w}\right)$. By Theorem 2, we know that $\operatorname{cost}_{G\left[V_{1 / 2}\right]}\left(S_{1 / 2}^{*}\right) \geq$ $\frac{1}{2} \cdot \widetilde{w}\left(V_{1 / 2}\right)$. Since $G\left[V_{1 / 2}\right]$ has degree bounded by $d$, and it is neither an odd cycle nor a complete graph, we can color it using $d$ colors [6]. Now, let $S_{1 / 2}$ be the subset of $V_{1 / 2}$ excluding the heaviest color class with respect to $\widetilde{w}$. We have

$$
\operatorname{cost}_{G\left[V_{1 / 2}\right]}\left(S_{1 / 2}^{*}\right) \geq \frac{1}{2} \cdot \widetilde{w}\left(V_{1 / 2}\right) \geq \frac{d}{2(d-1)} \cdot \widetilde{w}\left(S_{1 / 2}\right)=\frac{d}{2(d-1)} \cdot \operatorname{cost}_{G\left[V_{1 / 2}\right]}\left(S_{1 / 2}\right),
$$

and therefore, $V_{1} \cup S_{1 / 2}$ is $\left(2-\frac{2}{d}\right)$-approximate.

4.2. Planar graphs. We next use the technique of Baker [1] together with Theorem 2 to obtain a PTAS for Generalized Vertex Cover in planar graphs. The main idea is to first use the algorithm of Theorem 2 and then to break the planar subgraph $G\left[V_{1 / 2}\right]$ into a set of $k$-outerplanar graphs by removing a set of vertices from $G$ whose weight is at most $\widetilde{w}\left(V_{1 / 2}\right) / k$. Since $k$-outerplanar graphs have treewidth depending only on $k$, we can compute an optimal solution for each graph in the set of remaining $k$-outerplanar graphs. (See [24] for the definition of treewidth.) Furthermore, since $\widetilde{w}\left(V_{1 / 2}\right)$ is at most twice the cost of the optimum solution in $\left(G\left[V_{1 / 2}\right], \widetilde{w}\right)$ by Theorem 2, this removed set of vertices together with optimal solutions of the $k$-outerplanar graphs constitutes a $\left(1+\frac{2}{k}\right)$-approximate generalized vertex cover.

We begin with a formal definition of tree decompositions and treewidth.

Definition 2 (tree decomposition [24]). A tree decomposition of a graph $G=$ $(V, E)$ is a pair $(\mathcal{T}, \mathcal{X})$, where $\mathcal{X} \subseteq 2^{V}$ is a family of subsets of $V$ and $\mathcal{T}$ a tree over $\mathcal{X}$ satisfying the following conditions:

1. $\bigcup_{X \in \mathcal{X}} G[X]=G$, and

2. $\mathcal{X}_{v}=\{X \in \mathcal{X} \mid v \in X\}$ is connected in $\mathcal{T}$ for each $v \in V$.

The width of $\mathcal{T}$ is $\max _{X \in \mathcal{X}}|X|-1$. The treewidth of $G$ is the minimum width over all tree decompositions of $G$.

We solve Generalized VerTex Cover in graphs with bounded treewidth using a standard bottom-up dynamic programming approach.

Lemma 5. Generalized Vertex Cover can be solved in $2^{O(w)} \cdot n$ time in graphs of treewidth at most $w$.

Proof. We start with some notation. Let $R$ be an arbitrary root of $\mathcal{T}$, and let $\mathcal{T}(X)$ denote the subtree of $\mathcal{T}$ whose root is $X$. (In this notation $\mathcal{T}(R)=\mathcal{T}$.) Also, let $G_{X}$ be the subgraph of $G$ that is induced by the vertex set $\bigcup_{Y \in \mathcal{T}(X)} Y$.

Now, for any $X \in \mathcal{X}$ and any $S \subseteq X$, let $\Pi(X, S)$ denote the cost of an optimal generalized vertex cover $S^{*}$ in $\left(G_{X}, w\right)$ such that $S^{*} \cap X=S$. We compute $\Pi(X, S)$ in a bottom-up fashion. First, if $X$ is a leaf in $\mathcal{T}$, then $\Pi(X, S)$ can be computed directly. Otherwise, let $X_{0}$ be an internal node of $\mathcal{T}$, and let $X_{1}, X_{2}, \ldots, X_{d}$ be its 
children. Since $\Pi\left(X_{0}, S_{0}\right)$ is obtained by some vertex set $S^{*} \subseteq \bigcup_{Y \in \mathcal{T}(X)} Y$ such that $S_{0}=X_{0} \cap S^{*}$, we can compute $\Pi\left(X_{0}, S_{0}\right)$ using the following recurrence:

$\Pi\left(X_{0}, S_{0}\right)=\min _{S: S_{0} \subseteq S \subseteq \bigcup_{i=0}^{d} X_{i}}\left(\operatorname{cost}_{G\left[X_{0}\right]}\left(S_{0}\right)+\sum_{i=1}^{d}\left(\Pi\left(X_{i}, S \cap X_{i}\right)-\operatorname{cost}_{G\left[X_{0} \cap X_{i}\right]}\left(S_{0} \cap X_{i}\right)\right)\right)$.

The idea is that we add the cost of $S^{*}$ in $G_{X_{i}}$, for any $i=1, \ldots, d$, to the cost of $S^{*}$ in $G[X]$, and then we subtract the weights that were counted more than once. For example, when $d=1$, we add $\Pi\left(X_{1}, S \cap X_{1}\right)$ to the cost of $S_{0}$ in $G\left[X_{0}\right]$ and subtract the cost of $S_{0} \cap X_{1}$ in $G\left[X_{0} \cap X_{1}\right]$.

Note that the computation of $\Pi$ can be easily modified to computing a corresponding generalized vertex cover. Since one may assume without loss of generality that $\mathcal{T}$ is binary, the running time for computing an entry $\Pi\left(X_{0}, S_{0}\right)$ is $2^{O(w)}$. Hence, the total running time is $2^{O(w)} \cdot n$.

Corollary 4. Generalized Vertex Cover in planar graphs has a PTAS.

Proof. Given a planar embedding of a planar graph, the vertices on the exterior face are said to be at level 0 . After the removal of level 1 vertices, the vertices of the resulting exterior face are said to be in level 1 . Level $i$ vertices are the vertices that are contained in exterior face after the removal of vertices from level $0, \ldots, i-1$. We denote the set of level $i$ vertices by $L_{i}$. In these terms, a $k$-outerplanar graph has a planar embedding with $k$ layers.

Let $G$ be a planar graph, and let $G\left[V_{1 / 2}\right]$ be the planar subgraph obtained by Theorem 2. By the theorem, a PTAS on $\left(G\left[V_{1 / 2}\right], \widetilde{w}\right)$ would imply a PTAS on $(G, w)$. Let $k$ be some positive integer, and let $G_{i}, i \in\{0, \ldots, k\}$, be the graph obtained by removing layers $i+(k+1) j$, for $j=0,1, \ldots$, from $G\left[V_{1 / 2}\right]$. Since $G_{i}$ is $k$-outerplanar for every $i \in\{0, \ldots, k\}$, its tree width is $3 k-1$ [5], and therefore, we can compute an optimal generalized vertex cover $U_{i}^{*}$ in $G_{i}$ in polynomial time using Lemma 5 . Letting $U_{i}=U_{i}^{*} \cup \bigcup_{j} L_{i+(k+1) j}$, we have

$$
\begin{aligned}
\sum_{i} \operatorname{cost}_{G}\left(U_{i}\right) & =\sum_{i} \operatorname{cost}_{G_{i}}\left(U_{i}^{*}\right)+\sum_{i} w\left(L_{i}\right) \\
& \leq \sum_{i} \operatorname{opt}\left(G\left[V_{1 / 2}\right], \widetilde{w}\right)+2 \operatorname{opt}\left(G\left[V_{1 / 2}\right], \widetilde{w}\right) \\
& =(k+2) \cdot \operatorname{opt}\left(G\left[V_{1 / 2}\right], \widetilde{w}\right) .
\end{aligned}
$$

Hence, the solution with minimum weight among the $U_{i}$ 's is $\left(1+\frac{2}{k}\right)$-approximate. Finally, picking $k=\frac{2}{\epsilon}$, we obtain a $(1+\epsilon)$-approximation for every $\epsilon$.

4.3. General graphs. We now show that the $\left(2-\frac{\lg \lg n}{2 \lg n}\right)$-approximation algorithm of [3] can be extended to Generalized Vertex Cover, due to Theorem 2. The central component in the algorithm of [3] is given in the following lemma.

Lemma 6 (see [3]). There is a polynomial-time algorithm that, given a graph $G=(V, E)$, a weight function $w: V \rightarrow \mathbb{Q}^{\geq 0}$, and an integer $k$ such that

1. $|V| \geq(2 k-1)^{k}$, and

2. $G$ does not contain an odd cycle of length at most $2 k-1$, computes a vertex cover $C$ of $G$ with $w(C) \leq\left(1-\frac{1}{2 k}\right) w(V)$.

Another component we use is due to the local-ratio technique: We can remove odd cycles in a given instance $(G, w)$ of Generalized Vertex Cover at a relatively small cost to our approximation guarantee. We have the following lemma.

Lemma 7. Let $(G, w)$ be an instance of Generalized Vertex Cover, and let $C$ be an odd cycle in $G$ of size $2 t-1$, where $t \leq k$. Then the weight function $w_{\epsilon}$ 
which assigns $\epsilon$ to all vertices and edges in $C$ and 0 to all other vertices and edges is $\left(2-\frac{1}{k}\right)$-effective.

Proof. First, observe that no solution for $\left(G, w_{\epsilon}\right)$ may weigh more than $(2 t-1) \cdot \epsilon$, since $w_{\epsilon}(V)=(2 t-1) \cdot \epsilon$. Furthermore, since the weights are uniform, paying a penalty for an edge is never better than taking one of its endpoints, and so an optimal solution for $\left(G, w_{\epsilon}\right)$ must have a cost of at least $t \cdot \epsilon$. It follows that any solution for $\left(G, w_{\epsilon}\right)$ is $(2-1 / t)$-approximate, and since $t \leq k$, we get that any solution is $(2-1 / k)$ approximate, and the lemma follows.

Corollary 5. Generalized Vertex Cover can be approximated within a factor of $2-\frac{\lg \lg n}{2 \lg n}$.

Proof. Let $k$ be the smallest integer for which $(2 k-1)^{k} \geq|V|$. Note that $k$ can be computed in polynomial time. We first remove odd cycles of length at most $2 k-1$ from the input graph. This is done as follows: As long as there is an odd cycle $C$ in $G$ having positive weights on all its vertices and edges, we construct a weight function $w_{\epsilon}$ as in Lemma 7, which is $(2-1 / k)$-effective according to the lemma. We then continue searching in $\left(G, w-w_{\epsilon}\right)$. Lemma 2 ensures that a $(2-1 / k)$-approximate solution with respect to $\left(G, w-w_{\epsilon}\right)$ will also be $(2-1 / k)$-approximate with respect to $\left(G, w-w_{\epsilon}\right)$. Let $w^{\prime}$ denote the weight function that we obtain when no odd cycles having positive weights on all their vertices and edges are left. Also, let $G^{\prime}$ be the graph obtained by removing all zero $w^{\prime}$-weight vertices from $G$, along with all edges incident to them, and all zero $w^{\prime}$-weight edges. Clearly, if $U$ is a $(2-1 / k)$-approximate solution for $\left(G^{\prime}, w^{\prime}\right)$, then $U \cup V_{Z}$ is $(2-1 / k)$-approximate for $\left(G, w^{\prime}\right)$, where $V_{Z}$ is the set of all zero $w^{\prime}$-weight vertices in $G$.

We next use Theorem 2 on $\left(G^{\prime}, w^{\prime}\right)$ to obtain a partition of the vertices of $G^{\prime}$ into three subsets, $V_{1}^{\prime}, V_{0}^{\prime}$, and $V_{1 / 2}^{\prime}$, and to obtain the weight function $\widetilde{w}$. According to Theorem 2, an $\alpha$-approximation for $\left(G\left[V_{1 / 2}^{\prime}\right], \widetilde{w}\right)$ implies an $\alpha$-approximation on $\left(G^{\prime}, w^{\prime}\right)$. Since $G\left[V_{1 / 2}^{\prime}\right]$ satisfies the conditions of Lemma 6 , we can find a vertex cover $C$ for $G\left[V_{1 / 2}^{\prime}\right]$ such that $w(C) \leq(1-1 / 2 k) \cdot w(V)$. Due to the second condition in Theorem 2 , the subset $C$ is a $(2-1 / k)$-approximate solution for $\left(G\left[V_{1 / 2}^{\prime}\right], \widetilde{w}\right)$.

4.4. Fixed-parameter tractability. The Nemhauser-Trotter theorem has applications outside the world of approximation algorithms, most notably in the world of parameterized complexity. Chen, Kanj, and Jia [8] observed that this theorem gives a $2 k$ kernel for unweighted parameterized VERTEX COVER when the parameter is the number of vertices of the required vertex cover. We next note that, using Theorem 2, this straightforwardly extends to GENERALIZEd VERTEX Cover parameterized by the cost of the optimal solution.

Parameterized complexity deals with parameterized problems, whose instances are given together with a numeric parameter $k$ that encodes various structural properties of the input, e.g., solution size, maximum degree, and so forth. This allows a refined definition of tractable problems, where a tractable problem is now one with an algorithm running in $f(k) \operatorname{poly}(n)$ time, where $n$ is the instance size and $f$ is any computable function. FPT is the class of all parameterized problems with an $f(k) \operatorname{poly}(n)$ algorithm. A kernelization algorithm, or simply a kernel, is a commonly used technique for showing that a parameterized problem is in FPT. Formally, a kernel is a polynomial-time algorithm that transforms an instance $(I, k)$ to an instance $\left(I^{\prime}, k^{\prime}\right)$, with $\left|I^{\prime}\right|+k^{\prime} \leq f(k)$ for some computable function $f$, such that $(I, k)$ is a "yes"-instance if and only if $\left(I^{\prime}, k^{\prime}\right)$ is a "yes"-instance. It is easy to see that Theorem 2 gives exactly this when the parameter is taken as the cost of the solution. 
Corollary 6. Generalized Vertex Cover parameterized by the cost $k$ of the optimal solution has a $2 k$ kernel.

\section{REFERENCES}

[1] B. S. BAKER, Approximation algorithms for NP-complete problems on planar graphs, J. ACM, 41 (1994), pp. 153-180.

[2] R. B. YenudA, One for the price of two: A unified approach for approximating covering problems, Algorithmica, 27 (2000), pp. 131-144.

[3] R. B. YehudA AND S. Even, A local-ratio theorem for approximating the weighted vertex cover problem, Ann. Discrete Math., 25 (1985), pp. 27-46.

[4] R. B. YenUdA AND D. RAwitz, On the equivalence between the primal-dual schema and the local ratio technique, SIAM J. Discrete Math., 19 (2005), pp. 762-797.

[5] H. L. Bodlaender, Classes of graphs with bounded tree-width, Technical report RUU-CS-86-2, Utrecht University, The Netherlands, 1986.

[6] R. L. Brooks, On colouring the nodes of a network, Math. Proc. Cambridge Philos. Soc., 37 (1941), pp. 194-197.

[7] M. Charikar, S. Khuller, D. M. Mount, and G. Narasimhan, Algorithms for facility location problems with outliers, in 12th Annual ACM-SIAM Symposium on Discrete Algorithms, 2001, pp. 642-651.

[8] J. Chen, I. A. KAnJ, AND W. JiA, Vertex cover: Further observations and further improvements, J. Algorithms, 41 (2001), pp. 280-301.

[9] M. Chlebík And J. ChlebíKovÁ, Improvement of Nemhauser-Trotter Theorem and Its Applications in Parametrized Complexity, in Algorithm Theory-SWAT, 2004, Lecture Notes in Comput. Sci. 3111, Springer, New York, 2004, pp. 174-186.

[10] I. Dinur And S. SAFra, The importance of being biased, in Proceedings of the 34th ACM Symposium on the Theory of Computing, 2002, pp. 33-42.

[11] M. X. Goemans And D. P. Williamson, A general approximation technique for constrained forest problems, SIAM J. Comput., 24 (1995), pp. 296-317.

[12] R. Hassin And A. Levin, The minimum generalized vertex cover problem, ACM Trans. Algorithms, 2 (2006), pp. 66-78.

[13] R. HASSin AND A. TAMIR, Improved complexity bounds for location problems on the real line, Oper. Res. Lett., 10 (1991), pp. 395-402.

[14] D. S. Hochbaum, Approximation algorithms for the set covering and vertex cover problems, SIAM J. Comput., 11 (1982), pp. 555-556.

[15] D. S. Hochbaum, Efficient bounds for the stable set, vertex cover and set packing problems, Discrete Appl. Math., 6 (1983), pp. 243-254.

[16] D. S. Hochbaum, Solving integer programs over monotone inequalities in three variables: A framework of half integrality and good approximations, European J. Oper. Res., 140 (2002), pp. 291-321.

[17] K. Jain, M. Mahdian, E. Markakis, A. Saberi, and V. V. Vazirani, Greedy facility location algorithms analyzed using dual-fitting with factor-revealing LP, J. ACM, 50 (2003), pp. $795-824$.

[18] R. M. KARP, Reducibility among combinatorial problems, in Complexity of Computer Computations: Proceedings of a Symposium on the Complexity of Computer Computations, R. E. Miller and J. W. Thatcher, eds., Plenum Press, New York, 1972, pp. 85-103.

[19] J. Könemann, O. PAREKh, And D. SEgev, A unified approach to approximating partial covering problems, in Proceedings of the 14th European Symposium on Algorithms (ESA06), 2006, pp. 468-479.

[20] D. KöNIG, Graphs and matrices, Matematikai és Fizikai Lapok, 38 (1931), pp. 116-119 (in Hungarian).

[21] R. J. Lipton And R. E. TARJAn, A separator theorem for planar graphs, SIAM J. Appl. Math., 36 (1979), pp. $177-189$

[22] G. L. Nemhauser And L. E. Trotter, JR., Properties of vertex packing and independence system polyhedra, Math. Program., 6 (1974), pp. 48-61.

[23] G. L. Nemhauser and L. E. Trotter, JR., Vertex packings: Structural properties and algorithms, Math. Program., 8 (1975), pp. 232-248.

[24] N. Robertson and P. D. Seymour, Graph minors, II: Algorithmic aspects of tree-width, J. Algorithms, 7 (1986), pp. 309-322. 